\title{
Analysis of the High Resolution ECG with the Continuous Wavelet Transform
}

\author{
S Bunluechokchai, MJ English \\ University of Sussex, Brighton, UK
}

\begin{abstract}
Ventricular Late Potentials (VLPS) are low-level highfrequency signals that are usually found within the terminal part of the QRS complex from patients after Myocardial Infarction. They are used as a diagnostic indicator of a patient prone to Ventricular Tachycardia, which is a major cause of death.

In this study, the Continuous Wavelet Transform was used to analyse the High Resolution ECG. Then the total energy at different time intervals of the terminal region of the QRS complex was computed. In order to investigate what time interval gave the best performance for revealing VLPS to separate patients with VLPS from those without. Preliminary investigations show that some of the time intervals offered significant differences in the total energy between the two patient groups.
\end{abstract}

\section{Introduction}

Ventricular Late Potentials are low-amplitude, highfrequency signals that generally occur within the terminal part of the QRS complex and the beginning of the ST segment. It is supposed that VLPs are generated from a region of the damaged myocardium where the ventricular depolarisation is delayed. VLPs can be recorded with the High Resolution ECG (HRECG). They appear as fractionated microvolt signals on the body surface. From the studies in clinical cardiology, it has been shown that the occurrence of VLPs is prevalent in post-Myocardial Infarction (MI) patients at risk of developing Ventricular Tachycardia, which is one of the leading causes of sudden cardiac death. Hence the detection of VLPs has become a topic of interest in clinical cardiology for over three decades. Early diagnosis of heart disease by detecting VLPs might save a large number of patients' lives.

The standard method for detecting VLPs was proposed by Simson [1,2]. This method computes the parameter measurements obtained from the filtered signal-averaged ECG in the time domain. Many attempts have been made to study VLPs using time-frequency domain techniques, for example the Wigner Distribution (WD) and the Short Time Fourier Transform (STFT). The limitation of the WD is that it produces unwanted interference terms that do not reflect the original signal whereas the STFT has fixed time-frequency resolution that is not optimal for the analysis of non-stationary signals. VLPs are assumed to be non-stationary signals. The wavelet transform is proposed as an alternative technique and it can solve some of the limitations encountered in the WD and STFT.

In this study, the Continuous Wavelet Transform (CWT) was used to analyse the HRECG. The aim of this study was to investigate what time interval of the terminal region of the QRS complex showed the most difference between patients with VLPs and those without by computing the total energy obtained from the CWT.

\section{Data acquisition}

High Resolution ECG recordings were acquired from post-MI patients using orthogonal XYZ leads at a sampling rate of $1000 \mathrm{~Hz}$ and a resolution of 12 bits. The standard time domain method [1,2] was used to identify patients who showed the presence of VLPs. According to the standard method, the patient data set was divided into two groups, 20 patients with and 49 without VLPs. The results of the two patient groups were taken as a reference to validate the proposed method in this study.

\section{The continuous wavelet transform}

More recently, among time-frequency methods, the CWT seems to be a tool of choice for HRECG analysis $[3,4]$. The main advantage of the CWT is that it provides variable time frequency resolution. It is defined as:

$$
C W T(\tau, a)=\frac{1}{\sqrt{a}} \int_{-\infty}^{\infty} s(t) \psi\left(\frac{t-\tau}{a}\right) d t
$$

where $s(t)$ is the signal, $\psi(t)$ is the mother wavelet, scaled by $a$ and shifted by $\tau$. The scaling parameter $a$ can be viewed as the inverse of frequency. Dilating or contracting the wavelet changes frequencies of interest. The wavelet is contracted at smaller scales, corresponding to high frequencies, which can detect high frequency components of the signal whereas the wavelet is dilated at larger scales, relating to low frequencies, which can extract the low frequency components of the signal. This provides varying time-frequency resolution. Shifting the wavelet is to localise the signal in time. The CWT thus offers a good compromise between time localisation and frequency resolution. Further details 
about the CWT can be found in the literature $[5,6]$. It is obvious that the variable time frequency resolution characteristic of the CWT is well suited to the analysis of non-stationary signals containing short, high-frequency components. As VLPs are considered to be short-duration high-frequency signals, the CWT seems to be a promising technique for this task. Computation of the energy percentage of the HRECG with the CWT has been reported [7]. Because the CWT is computed in terms of scale instead of frequency, it produces a time-scale representation of the signal.

\section{HRECG analysis with the CWT}

In order to reveal and extract the informative energy of the low-level high-frequency VLPs with the CWT, it was necessary to process the VLPs with small scales corresponding to high frequencies. The CWT was applied to each averaged $\mathrm{XYZ}$ lead and then the vector magnitude CWT was formed from the three CWT magnitudes CWTX, CWTY, CWTZ as in Equation 2. The CWT of scales 3-20, in steps of 0.2 , using a Daubechies 2 mother wavelet, was investigated. Using scales 3-20 substantially helps avoid the high energy of the $\mathrm{R}$ wave, which greatly masks the low energy of the low level VLPs. The total energy of the QRS complex was normalised to unity.

$$
|C W T V|=\sqrt{\left(C W T X^{2}+C W T Y^{2}+C W T Z^{2}\right)}
$$

where $C W T V$ is the vector magnitude. $C W T X, C W T Y$, and $C W T Z$ are the CWT of the XYZ leads, respectively.

Summing all of the energies for all scales from 3-20 will give the total signal energy. The total signal energy was computed from the $C W T V$. The total energy obtained from the sum of all of the energies for scales 3-20 was calculated for 7 groups of different time intervals within the terminal part of the QRS complex and the early part of the ST segment for the patients with and without VLPS as follows:

1. Group 1: a group of 7 different time intervals starting from $10 \mathrm{~ms}$ before the end of the QRS complex, in steps of $10 \mathrm{~ms}$, up to $60 \mathrm{~ms}$ after the QRS end; thus these time intervals were as follows:

1.1 QRS end $-10+0 \mathrm{~ms}$

1.2 QRS end $+10 \mathrm{~ms}$

1.3 QRS end-10+20 ms

1.4 QRS end-10+30 ms

1.5 QRS end-10+40 ms

1.6 QRS end $-10+50 \mathrm{~ms}$

1.7 QRS end-10+60 ms

where "QRS end" is the end of the QRS complex.

"QRS end-10+0 ms" is the time interval from $10 \mathrm{~ms}$ before the QRS end to the QRS end.

"QRS end-10+60 $\mathrm{ms}$ " is the time interval from $10 \mathrm{~ms}$ before the end of the QRS complex to $60 \mathrm{~ms}$ after the QRS end.

2. Group 2: a group of 7 different time intervals starting from $20 \mathrm{~ms}$ before the end of the QRS complex, in steps of $10 \mathrm{~ms}$, up to $60 \mathrm{~ms}$ after the QRS end.
2.1 QRS end $-20+0 \mathrm{~ms}$
2.2 QRS end $-20+10 \mathrm{~ms}$
2.3 QRS end $+20 \mathrm{~ms}$
2.4 QRS end $-20+30 \mathrm{~ms}$
2.5 QRS end-20+40 ms
2.6 QRS end $-20+50 \mathrm{~ms}$
2.7 QRS end $-20+60 \mathrm{~ms}$

3. Group 3: a group of 7 different time intervals starting from $30 \mathrm{~ms}$ before the end of the QRS complex, in steps of $10 \mathrm{~ms}$, up to $60 \mathrm{~ms}$ after the QRS end.

4. Group 4: a group of 7 different time intervals starting from $40 \mathrm{~ms}$ before the end of the QRS complex, in steps of $10 \mathrm{~ms}$, up to $60 \mathrm{~ms}$ after the QRS end.

5. Group 5: a group of 7 different time intervals starting from $50 \mathrm{~ms}$ before the end of the QRS complex, in steps of $10 \mathrm{~ms}$, up to $60 \mathrm{~ms}$ after the QRS end.

6. Group 6: a group of 7 different time intervals starting from $60 \mathrm{~ms}$ before the end of the QRS complex, in steps of $10 \mathrm{~ms}$, up to $60 \mathrm{~ms}$ after the QRS end.

7. Group 7: a group of 6 different time intervals starting from the end of the QRS complex, in steps of $10 \mathrm{~ms}$, up to $60 \mathrm{~ms}$ after the QRS end.

\section{Results of the total energy}

Patients are classified as borderline patients if their measurement parameters computed from the filtered HRECG are very close to the criteria values of the Simson's standard method. The modified data set is a set of the patient data with the borderline patients removed. The complete data set consists of all patients.

The total energy was computed for each patient of the two groups at all of the different time intervals as mentioned above. The total energies for some selected time intervals are illustrated in Figure 1 . It can be observed that there is a significant difference between the patients with and without VLPs, especially in Figures 1c, and 1d. After the Wilcoxon Rank Sum test was applied to the total energy results for each of the different time intervals, the results showed that most of the time intervals, including the time interval between $40 \mathrm{~ms}$ before and $40 \mathrm{~ms}$ after the QRS end (Figure 1d), offered the same largest statistical difference between the two patient groups at the $99.9 \%$ level of confidence $(\mathrm{p}<0.0005)$ for the modified data set.

The total energy is relatively higher in the patients without VLPs than the total energy from those with. It can be seen that there is a big gap in the cluster of the total energies between the two patient groups in the modified data set (i.e. with the borderline cases not included). 

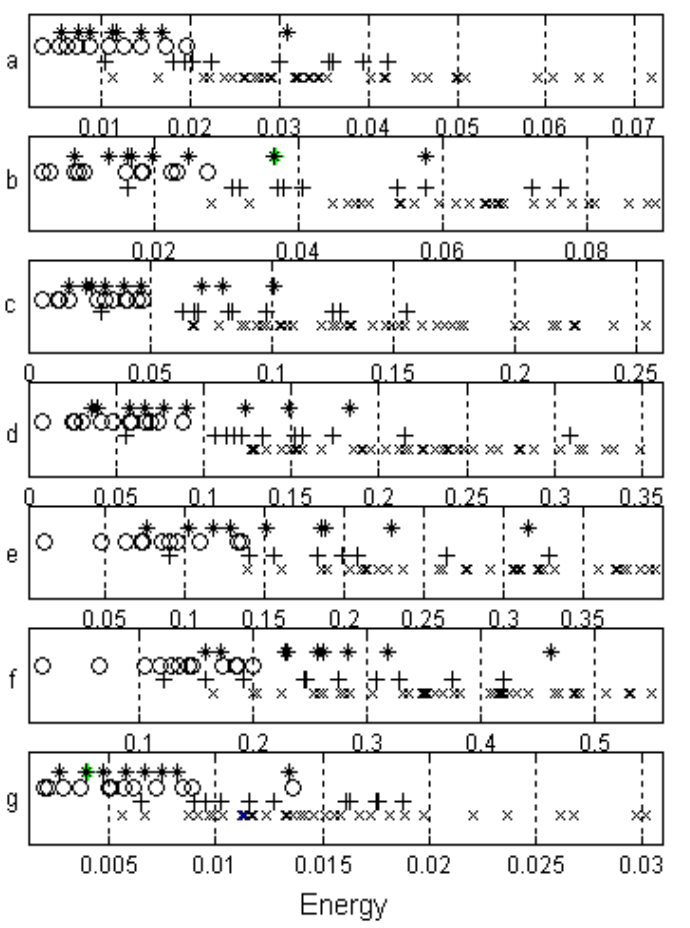

$$
\begin{array}{ll}
\times & \text { Patients without VPs } \\
0 & \text { Patients with VPs } \\
+ & \text { Patients without } \text { MPs but borderline } \\
* & \text { Patients with MPs but borderline }
\end{array}
$$

Figure 1. The total energy for QRS end-10+40 ms (a), QRS end-20+40 ms (b), QRS end-30+40 ms (c), QRS end $+40 \mathrm{~ms}(\mathrm{~d})$, QRS end-50+40 ms (e), QRS end-60+40 $\mathrm{ms}$ (f), QRS end+40 ms (g).

It quite clearly indicates that the total energy distributions of the patients without VLPs are shifted to the right of those with VLPs. It would be simply concluded that the observed difference is statistically significant $(\mathrm{p}<0.0005)$.

In Figure 1d, taking the results of the Simson method as a reference and empirically selecting a threshold level of 0.1 , sensitivity of $85 \%$ and specificity of $98 \%$ were obtained for the complete data set. When the borderline patients who were possibly misclassified by the Simson method were removed from the complete data set, and the sensitivity and specificity were recalculated, the resulting sensitivity and specificity were both $100 \%$ for the modified data set.

\section{Automatic detection of the QRS end}

The QRS end point used for computing the total energy in Section 4 was determined from visual inspection of the filtered vector magnitude in the time domain. In this section, determination of the end point of the QRS complex was automatically computed from the vector magnitude $C W T V$.
One of the distinct properties of the wavelet transform is that it has good time resolution for high frequencies. The wavelet transform can thus localise accurately in time high-frequency events of the signal. High-frequency characteristics of the signal can be best localised with small scales.

The averaged XYZ leads were filtered in a bidirectional mode with a $40 \mathrm{~Hz}$ high pass Butterworth filter and then the filtered XYZ leads were transformed by the CWT of scales of 1-8, using the Daubechies 2 wavelet. The vector magnitude CWT was formulated from Equation 2. Two contour plots of the $C W T V$ are plotted in Figure 2 for a patient without VLPs (a) and a patient with (b). The dotted line marks the filtered QRS end point.

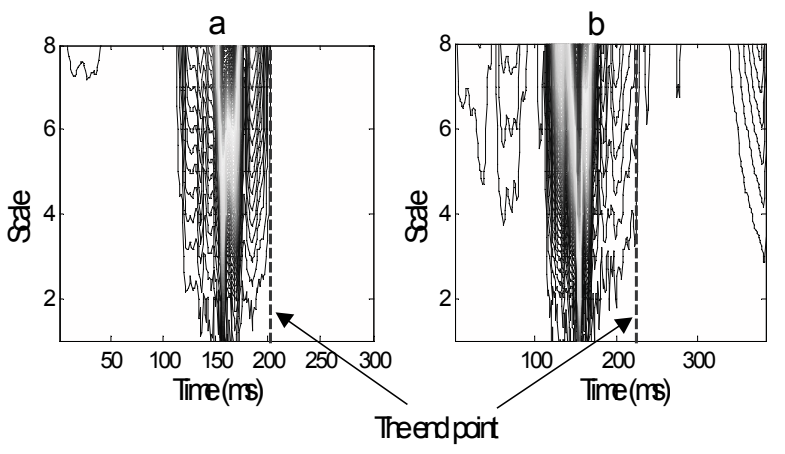

Figure 2. The QRS end point with the CWT contour for a patient without (a) and a patient with VLPs (b).

The end of the QRS complex was calculated from the $C W T V$ by the proposed automatic algorithm. The algorithm to identify the end point of the QRS complex was based on the mean noise level of a $50 \mathrm{~ms}$ segment. The mean noise level was computed from a region of the signal where cardiac activity is not present. The end of the QRS complex was automatically detected by searching the signal with a 3 sample segment for the mean of the segment above the threshold level. The midpoint of the 3 sample segment was defined as the QRS end point. The threshold level was computed from the mean noise level at the CWT scale of 6 . Once the automated end point of the QRS complex was obtained, the total energy was computed at the time interval from $40 \mathrm{~ms}$ before this automated end point to $40 \mathrm{~ms}$ after.

Results of the automated total energy for the two patient groups showed sensitivity of $80 \%$ and specificity of $96 \%$ for the complete data set, sensitivity and specificity of $100 \%$ for the modified data set.

\section{Discussion and conclusions}

VLPs are usually observed in the terminal region of the QRS complex and the beginning portion of the ST segment. The different time intervals for computing the total energy were tested on real data recordings to investigate what time interval offered the greatest 
statistical difference between the two patient groups and then the sensitivity and specificity of the results of the total energy were computed. The results showed that most of the investigated time intervals provided the same largest statistical difference between patients with and without VLPs. One of the most significantly different regions for the total energy was computed from $40 \mathrm{~ms}$ before the end of the QRS complex to $40 \mathrm{~ms}$ after. In this study, the time interval between $40 \mathrm{~ms}$ before the end of the QRS complex and $40 \mathrm{~ms}$ after was explored further by computing the total energy from the automated QRS end.

The results of the automated energy showed slightly lower sensitivity and specificity than those obtained from the total energy computed from the inspected QRS end point. This small error in sensitivity and specificity may be due to high background noise of some data recordings. However, the automatic QRS end algorithm was proposed to use the CWT for the detection of the QRS end point in the time-scale domain. In addition, the preliminary results in this study showed the possibility of detecting VLPs with total energy to differentiate between patients with and without VLPs. Patients without VLPs tended to have higher energy than those with. Ventricular depolarisation of the damaged myocardium is delayed and possibly partly obstructed, thus resulting in a decrease in the total energy of the QRS complex, whereas the ventricular depolarisation of the normal heart is homogeneous and synchronous in action, thus resulting in the higher energy.

\section{Acknowledgement}

The first author of this study was supported by a scholarship from the Thai government.

\section{References}

[1] Simson MB. Use of signals in the terminal QRS complex to identify patients with ventricular tachycardia after myocardial infarction. Circulation 1981;64(2):235-242.

[2] Breithardt G et al. Standards for analysis of ventricular late potentials using high resolution or signal-averaged electrocardiography - A statement by a Task Force Committee between the European Society of Cardiology, the American Heart Association and the American College of Cardiology. European Heart Journal 1991;12:473-480.

[3] Jones DL, Touvannas JS, Lander P, Albert DE. Advanced Time-frequency Methods for Signal Averaged ECG Analysis. Journal of Electrocardiology 1992;25:188-194.

[4] Khadra L, Dickhaus H, Lipp A. Representations of ECG late potentials in the time frequency plane. Journal of Medical Engineering and Technology 1993;17:228-231.

[5] Bentley PM, McDonnell JTE. Wavelet transforms: an introduction. IEE Electronics and Communication Engineering Journal 1994;175-186.

[6] Rioul O, Vetterli M. Wavelets and Signal Processing. IEEE Signal Processing Magazine 1991;14-38.

[7] Spaargaren A, English MJ. Analysis of the Signal Averaged ECG in the Time-Frequency domain. IEEE Computers in Cardiology 1999;26:571-574.

Address for correspondence.

Dr. M.J. English

Department of Engineering \& Design

University of Sussex

Brighton BN1 9QT

UK

m.j.english@sussex.ac.uk 